\title{
SOME REMARKS ON INDICES OF HOLOMORPHIC VECTOR FIELDS
}

\author{
Marco Brunella
}

\begin{abstract}
One can associate several residue-type indices to a singular point of a two-dimensional holomorphic vector field. Some of these indices depend also on the choice of a separatrix at the singular point. We establish some relations between them, especially when the singular point is a generalized curve and the separatrix is the maximal one. These local results have global consequences, for example concerning the construction of logarithmic forms defining a given holomorphic foliation.
\end{abstract}

Let $v$ be a holomorphic vector field defined on a neighborhood of $0 \in \mathbf{C}^{2}$ and having there an isolated singularity. Baum and Bott have associated to such a singular point two types of indices [BB], defined through residues of suitable meromorphic 2-forms. One of these indices is simply the Poincaré-Hopf index of $v$ at 0 , and so it is an integer positive number which gives the multiplicity of the singular point. The other one has a more subtle meaning, and we shall call it the Baum-Bott index, $B B(v, 0)$.

Let $S$ be a separatrix of $v$ at 0 , i.e. a holomorphic curve invariant by $v$ and containing 0 (there are always separatrices, see $[\mathbf{C S}]$ ). Camacho and $\mathrm{Sad}$, and later Lins Neto and Suwa, have defined an index $C S(v, S, 0)$ which, roughly speaking, represents the intersection index of the trajectories of $v$ with the separatrix $S[\mathbf{C s}],[\mathbf{L N}],[\mathbf{S u}]$. Gomez-Mont, Seade and Verjovsky have defined another index $G S V(v, S, 0)$ which is a sort of Poincaré-Hopf index of the restriction of $v$ to $S$ [GSV].

Aim of this paper is to underline some simple properties of these indices, and in particular to prove a relation between them for a rather general class of isolated singularities: the class of generalized curves [CLS], that is nondicritical singularities whose resolution does not contain saddle-nodes. In $[\mathbf{C L S}]$ it is proven that the Poincaré-Hopf index of such a singularity coincides with the Milnor number of the union of all the separatrices $S$ (which is still a separatrix, by nondicriticalness). Here we prove that the Baum-Bott index also can be "localized" near $S$. 
Theorem. Let $v$ be a holomorphic vector field near $0 \in \mathbf{C}^{2}, v(0)=0$, and suppose that the singularity at 0 is a generalized curve. Let $S$ be the union of all the separatrices of $v$ at 0 . Then

$$
\begin{aligned}
B B(v, 0) & =C S(v, S, 0) \\
G S V(v, S, 0) & =0 .
\end{aligned}
$$

This result is not so strange. The Baum-Bott index is something like a "mean intersection index at 0" of the leaves of the foliation $\mathcal{F}$ generated by $v$, whereas the Camacho-Sad index is related to the "intersection index at 0 " of the leaves of $\mathcal{F}$ with $S$. The leaves composing $S \backslash(0)$ are the only leaves which go "directly" to 0 , hence a relation between $B B(v, 0)$ and $C S(v, S, 0)$ is not surprising: two leaves both different from separatrices do not "intersect at 0 ". The actual proof is obtained by desingularization (the theorem is elementary for simple singularities, different from saddle-nodes; and it is false for saddle-nodes), and by the analysis of the variation of the indices under blow-ups. During the proof, the notion of nondicritical separatrix will naturally emerge.

In the last section of the paper we will see some global consequences of these types of results. We also include several remarks about $B B, C S$, and $G S V$ which are not strictly necessary to the proof of the theorem above, but which may be useful for a geometric understanding of these indices.

\section{The Baum-Bott index}

Let $\mathcal{F}$ be a holomorphic foliation with isolated singularities on a complex surface $X$. Let $p \in X$ be a singular point of $\mathcal{F}$; near $p$ the foliation is given either by a holomorphic vector field $v=F(z, w) \frac{\partial}{\partial z}+G(z, w) \frac{\partial}{\partial w}$ or by a holomorphic 1-form $\omega=F(z, w) d w-G(z, w) d z$. Here $(z, w)$ are local coordinates centered at $p$ and $F, G$, are holomorphic functions with $F^{-1}(0) \cap G^{-1}(0)=\{(0,0)\}$.

Let $J(z, w)$ be the Jacobian matrix of $(F, G)$ at $(z, w)$, then following $[\mathbf{B B}]$ we can defined two indices:

$$
\begin{aligned}
& P H(\mathcal{F}, p)=\operatorname{Res}_{(0,0)}\left\{\frac{\operatorname{det} J}{F \cdot G} d z \wedge d w\right\} \\
& B B(\mathcal{F}, p)=\operatorname{Res}_{(0,0)}\left\{\frac{(\operatorname{tr} J)^{2}}{F \cdot G} d z \wedge d w\right\}
\end{aligned}
$$

(see $[\mathbf{G H}]$ for the background concerning residues). These indices are well defined (i.e. they depend only on the conjugacy class of the germ of 
$\mathcal{F}$ at $p$ ), and they are easily computed when $p$ is non degenerate, that is when $J(0,0)$ is invertible: if $\lambda, \mu$ are the two eigenvalues of $J(0,0)$ then

$$
\begin{aligned}
& P H(\mathcal{F}, p)=\frac{\operatorname{det} J(0,0)}{\operatorname{det} J(0,0)}=1 \\
& B B(\mathcal{F}, p)=\frac{(\operatorname{tr} J(0,0))^{2}}{\operatorname{det} J(0,0)}=2+\frac{\lambda}{\mu}+\frac{\mu}{\lambda} .
\end{aligned}
$$

The index $P H(\mathcal{F}, p)$ is nothing else that the Poincaré-Hopf index of $v$ at $p$, hence it coincides with the multiplicity of the singular point and it can be also computed as a degree of a suitable map $[\mathbf{G H}]$. It follows from this its topological invariance $[\mathbf{C L S}]$. The index $B B(\mathcal{F}, p)$ has no meaning at the algebraic topology level, but it can be computed in the following way, à la Godbillon-Vey. On a pointed neighborhood $U^{*}=U \backslash\{p\}$ of $p$ we may find a complex valued smooth 1 -form $\beta$, of type $(1,0)$, such that

$$
d \omega=\beta \wedge \omega .
$$

For example, we may set $\beta=\frac{F_{z}+G_{w}}{|F|^{2}+|G|^{2}}(\bar{F} d z+\bar{G} d w)$. Using the cohomological interpretation of residues $[\mathbf{G H}]$ it is easy to verify that

$$
B B(\mathcal{F}, p)=\frac{1}{(2 \pi i)^{2}} \int_{\mathbf{S}^{3}} \beta \wedge d \beta
$$

where $\mathbf{S}^{3}$ in a small sphere around $p$, oriented as a boundary of a small ball containing $p$. It is in fact sufficient that $\beta$ is defined on a neighborhood of such a sphere.

If $\mathcal{F}$ is a foliation on a compact surface $X$ then the sum of Baum-Bott indices at singular points is equal to $c_{1}^{2}\left(N_{\mathcal{F}}\right)$, where $N_{\mathcal{F}} \in H^{1}\left(X, \mathcal{O}^{*}\right)$ is the normal bundle of $\mathcal{F}$ (which can be defined even in presence of singularities, $[\mathbf{G M}],[\mathbf{B r}])$. This is the Baum-Bott formula $[\mathbf{B B}]$, which can be straightforwardly generalized to the following situation. Without assuming the compactness of $X$, we consider a relatively compact domain $Y \subset X$ with $\partial Y$ smooth and disjoint from the singular set of $\mathcal{F}, \operatorname{Sing}(\mathcal{F})$. We assume that $N_{\mathcal{F}}$ is holomorphically trivial near $\partial Y$ (even if this is not completely necessary, the topological triviality would be sufficient), hence $\mathcal{F}$ near $\partial Y$ is given by a nonsingular holomorphic 1-form $\omega$ and we still can construct a smooth $(1,0)$-form $\beta$ near $\partial Y$ such that $d \omega=\beta \wedge \omega$. Then we define

$$
B B(\mathcal{F}, \partial Y)=\frac{1}{(2 \pi i)^{2}} \int_{\partial Y} \beta \wedge d \beta
$$

with $\partial Y$ oriented as boundary of $Y$, and of course this number does not depend on the involved choices. The triviality of $N_{\mathcal{F}}$ near the boundary allows also to define $c_{1}^{2}\left(N_{\mathcal{F}}\right) \in \mathbf{Z}$ (here $\left.\mathcal{F}=\left.\mathcal{F}\right|_{Y}\right)$. 
Proposition 1 [BB].

$$
B B(\mathcal{F}, \partial Y)=\sum_{p \in \operatorname{Sing}(\mathcal{F}) \cap Y} B B(\mathcal{F}, p)-c_{1}^{2}\left(N_{\mathcal{F}}\right)
$$

We shall use this formula in the process of desingularization.

Let us return to the local situation, and let $S$ be a separatrix of $\mathcal{F}$ at $p$, that is a holomorphic curve on a neighborhood of $p$, containing $p$ and invariant by $\mathcal{F}$. It is not assumed that $S$ is smooth nor irreducible at $p$. Using the 1-form $\beta$ we may define the following index $[\mathbf{K S}]$ :

$$
\operatorname{Var}(\mathcal{F}, S, p)=\frac{1}{2 \pi i} \int_{\partial S} \beta
$$

where $\partial S=S \cap \mathbf{S}^{3}$ and $\mathbf{S}^{3}$ is again a small sphere around $p ; \partial S$ is oriented as a boundary of $S \cap \mathbf{B}^{4}$, with $\mathbf{B}^{4}$ a small ball containing $p$. To give a consistent definition it is in fact sufficient that $\beta$ is defined only on a neighborhood of $\partial S$, and that $d \omega=\beta \wedge \omega$ holds only at points of $\partial S$.

Suppose now that $S \subset X$ is a compact holomorphic curve invariant by the foliation $\mathcal{F}$.

Proposition $2[\mathrm{KS}]$.

$$
\sum_{p \in \operatorname{Sing}(\mathcal{F}) \cap S} \operatorname{Var}(\mathcal{F}, S, p)=c_{1}\left(N_{\mathcal{F}}\right) \cdot S
$$

Propositions 1 and 2 are manifestations of the same principle. We may find a covering $\mathcal{U}=\left\{U_{j}\right\}$ of $X$, holomorphic 1-forms with isolated singularities $\omega_{j} \in \Omega^{1}\left(U_{j}\right)$, smooth $(1,0)$-forms $\beta_{j} \in A^{(1,0)}\left(U_{j}\right)$, such that:

i) $\left.F\right|_{U_{j}}$ is defined by $\omega_{j}$ (hence on $U_{j} \cap U_{i}$ one has $\omega_{i}=g_{i j} \omega_{j}$ and $\left\{g_{i j}\right\}$ is a $\mathcal{O}^{*}$-cocycle defining $N_{\mathcal{F}}$ ).

ii) $d \omega_{j}=\beta_{j} \wedge \omega_{j}$ on $U_{j} \backslash V_{j}$, where $V_{j}$ is a small neighborhood of $\operatorname{Sing}(\mathcal{F}) \cap U_{j}$.

iii) $\beta_{i}-\beta_{j}=\frac{d g_{i j}}{g_{i j}}$ on $U_{i} \cap U_{j}$.

Then the 2-form $\Theta$ locally given by $\Theta=\frac{1}{2 \pi i} d \beta_{j}$ represents (in the De Rham sense) the first Chern class of $N_{\mathcal{F}}$, hence $c_{1}^{2}\left(N_{\mathcal{F}}\right)=\int_{X} \Theta \wedge \Theta$, $c_{1}\left(N_{\mathcal{F}}\right) \cdot S=\int_{S} \Theta$, and Propositions 1 and 2 follow. 


\section{The Camacho-Sad index}

Let us consider again a separatrix $S$ at $p \in X$. Let $f$ be a holomorphic function on a neighborhood of $p$ and defining $S: S=\{f=0\}$. We may assume that $f$ is reduced, i.e. $d f \neq 0$ outside $p$. Then $[\mathbf{L N}]$, $[\mathbf{S u}]$ there are functions $g, k$ and a 1 -form $\eta$ on a neighborhood of $p$ such that

$$
g \omega=k d f+f \eta
$$

and moreover $k$ and $f$ are prime, i.e. $k \neq 0$ on $S^{*}=S \backslash\{p\}$. Remark that on $S$ we have $g \omega=k d f$, and the nonvanishing of $k$ and $d f$ on $S^{*}$ guarantees that also $g \neq 0$ on $S^{*}$.

The Camacho-Sad index $[\mathbf{C S}],[\mathbf{L N}]$, $[\mathbf{S u}]$ is defined as

$$
C S(\mathcal{F}, S, p)=-\frac{1}{2 \pi i} \int_{\partial S} \frac{1}{k} \eta .
$$

For example, let $\mathcal{F}$ be generated by $v(z, w)=z(\lambda+\cdots) \frac{\partial}{\partial z}+w(\mu+\cdots) \frac{\partial}{\partial w}$, where the dots denote terms vanishing at $(0,0)=p$ and $\lambda, \mu \neq 0$. Let $S_{1}=\{z=0\}, S_{2}=\{w=0\}$. Then $[\mathbf{S u}]$

$$
\begin{aligned}
C S\left(\mathcal{F}, S_{1}, p\right) & =\frac{\lambda}{\mu} \\
C S\left(\mathcal{F}, S_{2}, p\right) & =\frac{\mu}{\lambda} \\
C S\left(\mathcal{F}, S_{1} \cup S_{2}, p\right) & =2+\frac{\lambda}{\mu}+\frac{\mu}{\lambda} .
\end{aligned}
$$

This example shows that $C S(\mathcal{F}, \cdot, p)$ is not additive on the set of separatrices (whereas $\operatorname{Var}(\mathcal{F}, \cdot, p)$ is). More precisely [Su], if $S=S_{1} \cup S_{2}$ then

$$
C S(\mathcal{F}, S, p)=C S\left(\mathcal{F}, S_{1}, p\right)+C S\left(\mathcal{F}, S_{2}, p\right)+2\left(S_{1} \cdot S_{2}\right)_{p}
$$

where $\left(S_{1} \cdot S_{2}\right)_{p}$ is the local intersection number of $S_{1}$ and $S_{2}$ at $p$.

If $S \subset X$ is a compact holomorphic curve invariant by $\mathcal{F}$, one obtains the following formula (see $[\mathbf{K S}]$ for a direct proof, without desingularization).

Proposition $3[\mathbf{C S}],[\mathbf{L N}]$, [Su].

$$
\sum_{p \in \operatorname{Sing}(\mathcal{F}) \cap S} C S(\mathcal{F}, S, p)=S \cdot S .
$$

Remark the consistency of this formula with the above discussion around the non additivity of $C S(\mathcal{F}, \cdot, p)$. 


\section{The Gomez-Mont-Seade-Verjovsky index}

We continue with hypothesis and notations of the previous section. Define

$$
G S V(\mathcal{F}, S, p)=\frac{1}{2 \pi i} \int_{\partial S} \frac{g}{k} d\left(\frac{k}{g}\right) .
$$

Let us verify that this index coincides with the one introduced in [GSV]. Let us denote by $J$ the isomorphism from 1 -forms to vector fields induced by a nonsingular holomorphic 2-form near $p$. The holomorphic vector field $J(\omega)$ defines $\mathcal{F}$ and it can be decomposed as a sum of two meromorphic vector fields:

$$
J(\omega)=\frac{k}{g} J(d f)+\frac{f}{g} J(\eta)=v_{1}+v_{2}
$$

Observe that: i) on $S$ we have $J(\omega)=v_{1}$; ii) $v_{1}$ is tangent to $S_{\epsilon}=\{f=$ $\epsilon\}, \epsilon \neq 0$ small; iii) $\left.v_{1}\right|_{S_{\epsilon}}$ has poles in correspondence of $S_{\epsilon} \cap\{g=0\}$ and zeroes in correspondence of $S_{\epsilon} \cap\{k=0\}$. It is then clear that the index defined in $[\mathbf{G S V}]$ coincides with the difference between the number of zeroes and the number of poles of $\left.v_{1}\right|_{S_{\epsilon}}$, i.e. with $\frac{1}{2 \pi i} \int_{\partial S_{\epsilon}} \frac{d k}{k}-\frac{d g}{g}=$ $\frac{1}{2 \pi i} \int_{\partial S} \frac{g}{k} d\left(\frac{k}{g}\right)=G S V(\mathcal{F}, S, p)$.

Taking the example of the previous section, one finds

$$
\begin{aligned}
G S V\left(\mathcal{F}, S_{1}, p\right)=G S V\left(\mathcal{F}, S_{2}, p\right) & =1, \\
G S V\left(\mathcal{F}, S_{1} \cup S_{2}, p\right) & =0
\end{aligned}
$$

and, as $C S$, also $G S V(\mathcal{F}, \cdot, p)$ is not additive on the set of separatrices. From Proposition 5 below, or by a direct computation as in $[\mathbf{S u}]$ for $C S$, one finds that if $S=S_{1} \cup S_{2}$ then

$$
G S V(\mathcal{F}, S, p)=G S V\left(\mathcal{F}, S_{1}, p\right)+G S V\left(\mathcal{F}, S_{2}, p\right)-2\left(S_{1} \cdot S_{2}\right)_{p}
$$

If $S \subset X$ is a compact curve invariant by $\mathcal{F}$ then one has the following formula (in $[\mathbf{K S}]$ it is formulated, in an equivalent way, using the Schwarz index, whose difference with the $G S V$ index is the Milnor number of $S$ at $p)$.

Proposition 4 [Br], [KS].

$$
\sum_{p \in \operatorname{Sing}(\mathcal{F}) \cap S} G S V(\mathcal{F}, S, p)=c_{1}\left(N_{\mathcal{F}}\right) \cdot S-S \cdot S .
$$

In fact, Proposition 4 follows also from Propositions 2 and 3 and the following relation between the three indices $\operatorname{Var}, C S, G S V$. 
Proposition 5. If $S$ is any separatrix at $p$ then

$$
\operatorname{Var}(\mathcal{F}, S, p)=G S V(\mathcal{F}, S, p)+C S(\mathcal{F}, S, p)
$$

Proof: Observe that, at points of $\partial S$ :

$$
\begin{aligned}
\omega & =\frac{k}{g} d f \\
d \omega & =\left(d\left(\frac{k}{g}\right)-\frac{1}{g} \eta\right) \wedge d f
\end{aligned}
$$

hence $d \omega=\left(\frac{g}{k} d\left(\frac{k}{g}\right)-\frac{1}{k} \eta\right) \wedge \omega$ and consequently

$$
\begin{aligned}
\operatorname{Var}(\mathcal{F}, S, p)=\frac{1}{2 \pi i} \int_{\partial S}\left(\frac{g}{k} d\left(\frac{k}{g}\right)\right. & \left.-\frac{1}{k} \eta\right) \\
& =G S V(\mathcal{F}, S, p)+C S(\mathcal{F}, S, p) .
\end{aligned}
$$

The $G S V$ index is, of course, an integer number, but it may be negative; this is the obstruction to the positive solution to "Poincaré problem" [Car], [Br], because in $\mathbf{C} P^{2}$ the inequality $c_{1}\left(N_{\mathcal{F}}\right) \cdot S \geq S \cdot S$ means $\operatorname{deg}(\mathcal{F})+2 \geq \operatorname{deg}(S)$. Here, after some definitions, we give a simple condition (related to $[\mathbf{C a r}]$ ) that implies the nonnegativity of $G S V$.

We recall that the singular point $p$ is nondicritical if $\mathcal{F}$ has only a finite number of separatrices at $p$. This means that if $\pi: \tilde{X} \rightarrow X$ is the desingularization of $\mathcal{F}$ at $p$ then $\pi^{-1}(p)$ is entirely $\tilde{\mathcal{F}}$-invariant [CLS]. More generally, we shall say that a separatrix $S$ of $\mathcal{F}$ at $p$ is nondicritical if there is a sequence of blow-ups $\pi: \hat{X} \rightarrow X$, based at $p$, such that:

i) $\pi$ is a resolution of $S$, i.e. $\hat{S}=\pi^{-1}(S)$ is a curve with only normal crossing singularities;

ii) $\pi^{-1}(p)$ is $\hat{\mathcal{F}}$-invariant.

For instance, if $p$ is a nondicritical singular point of $\mathcal{F}$ then any separatrix of $\mathcal{F}$ at $p$ is nondicritical (take $\pi=$ resolution of $\mathcal{F}$ in the above definition). At the opposite case, if either $S$ is smooth at $p$ or $S$ has a normal crossing singular point at $p$ then $S$ is certainly nondicritical, independently on $\mathcal{F}$ (take $\pi=$ identity in the above definition).

Proposition 6. If $S$ is a nondicritical separatrix at $p$ then

$$
G S V(\mathcal{F}, S, p) \geq 0 .
$$


Proof: We shall prove the more general inequality

$$
G S V\left(\mathcal{F}, S^{\prime}, p\right) \geq\left(S^{\prime} \cdot S^{\prime \prime}\right)_{p}
$$

where $S^{\prime} \subset S$ is a union of irreducible components of $S$ and $S^{\prime \prime} \subset S$ is the union of those irreducible components which are not in $S^{\prime}$. Let us consider a function $f:(X, p) \rightarrow(\mathbf{C}, 0)$ such that $S=f^{-1}(0)$, but only in the set theoretic sense: if $S_{1}, \ldots, S_{N}$ are the irreducible components of $S$ and $f_{1}, \ldots, f_{N}$ their reduced equations, then $f=f_{1}^{p_{1}} \cdot \ldots \cdot f_{N}^{p_{N}}$, with $p_{j}$ positive integer numbers. As in $[\mathbf{C L S}]$, the idea is to compare $\mathcal{F}$ with the foliation $\mathcal{G}_{f}$ given by the level curves of $f$, i.e. by the 1 -form $f \cdot \sum_{j=1}^{N} p_{j} \frac{d f_{j}}{f_{j}}$ (remark that if $S$ is smooth then $\mathcal{G}_{f}$ is nonsingular, and if $S$ has a normal crossing singular point then $\mathcal{G}_{f}$ is linearizable). We denote by $\operatorname{ord}(\cdot, p)$ the order of a foliation at $p$, i.e. the vanishing order at $p$ of a 1-form generating the foliation near $p$.

\section{Claim.}

i) $\operatorname{ord}(\mathcal{F}, p) \geq \operatorname{ord}\left(\mathcal{G}_{f}, p\right)$

ii) $\operatorname{GSV}\left(\mathcal{F}, S^{\prime}, p\right) \geq G S V\left(\mathcal{G}_{f}, S^{\prime}, p\right)$.

This claim is proven by induction on the (minimal) number $n$ of blowups appearing in the definition of nondicritical separatrix.

$n=0$ : easy verification, left to the reader.

$n-1 \mapsto n$ : let $\pi: \tilde{X} \rightarrow X$ be a blow-up at $p, D=\pi^{-1}(p)$ its exceptional divisor, $\tilde{S}_{j}$ the strict transform of $S_{j}$ by $\pi, \tilde{\mathcal{F}}=\pi^{*}(\mathcal{F})$, $\tilde{\mathcal{G}}_{f}=\pi^{*}\left(\mathcal{G}_{f}\right)$. The curve $D$ is $\tilde{\mathcal{F}}$-invariant, by nondicriticalness of $S$, and clearly also $\tilde{\mathcal{G}}_{f}$-invariant. Each singular point $q$ at $\tilde{\mathcal{G}}_{f}$ on $D$ is also a singular point of $\tilde{\mathcal{F}}$; near such a point $\tilde{\mathcal{G}}_{f}$ is given by the levels of $\tilde{f}=f \circ \pi$, and $\tilde{f}^{-1}(0)$ is a nondicritical separatrix of $\tilde{\mathcal{F}}$ at $q$. The divisor $D$ is an irreducible component of this separatrix, hence, by induction hypothesis,

$$
\operatorname{GSV}(\tilde{\mathcal{F}}, D, q) \geq G S V\left(\tilde{\mathcal{G}}_{f}, D, q\right)
$$

The same inequality holds if $q \in \operatorname{Sing}(\tilde{\mathcal{F}}) \backslash \operatorname{Sing}\left(\tilde{\mathcal{G}}_{f}\right)$, because in that case the left hand side index is at least 1 and the right hand side index is 0 . Now, using Proposition 4 and the fact that $\operatorname{ord}(\mathcal{F}, p)=c_{1}\left(N_{\tilde{\mathcal{F}}}\right) \cdot D$ we obtain 


$$
\operatorname{ord}(\mathcal{F}, p)=-1+\sum_{q \in D \cap \operatorname{Sing}(\tilde{\mathcal{F}})} G S V(\tilde{\mathcal{F}}, D, q)
$$

and similarly

$$
\operatorname{ord}\left(\mathcal{G}_{f}, p\right)=-1+\sum_{q \in D \cap \operatorname{Sing}\left(\tilde{\mathcal{G}}_{f}\right)} G S V\left(\tilde{\mathcal{G}}_{f}, D, q\right) .
$$

Hence the first desired inequality $\operatorname{ord}(\mathcal{F}, p) \geq \operatorname{ord}\left(\mathcal{G}_{f}, p\right)$ follows from $G S V(\tilde{\mathcal{F}}, D, q) \geq G S V\left(\tilde{\mathcal{G}}_{f}, D, q\right)$.

Let now $m_{j}$ be the order of $S_{j}$ at $p$, so that $\tilde{f}_{j}=f_{j} \circ \pi$ vanishes on $D$ with order $m_{j}$. Set $M=\operatorname{ord}(\mathcal{F}, p)$, so that $\tilde{\omega}=\pi^{*} \omega$ vanishes on $D$ with order $M$. If $q_{j} \in D$ is the unique intersection point between $\tilde{S}_{j}$ and $D$, then near $q_{j}$ we may write

$$
\begin{gathered}
\tilde{\omega}=t^{M} \omega_{0} \\
\tilde{f}_{j}=t^{m_{j}} f_{0}
\end{gathered}
$$

where $t$ is a local equation of $D, \omega_{0}$ has an isolated zero at $q_{j}, f_{0}$ is a reduced equation of $\tilde{S}_{j}$. The usual decomposition of $\omega$,

$$
g \omega=k d f_{j}+f_{j} \eta
$$

implies

$$
\tilde{g} t^{M} \omega_{0}=\tilde{k} t^{m_{j}} d f_{0}+f_{0}\left(m_{j} t^{m_{j}-1} \tilde{k} d t+t^{m_{j}} \tilde{\eta}\right) .
$$

Hence $G S V\left(\tilde{\mathcal{F}}, \tilde{S}_{j}, q_{j}\right)$ is given by

$$
\begin{aligned}
G S V\left(\tilde{\mathcal{F}}, \tilde{S}_{j}, q_{j}\right) & =\frac{1}{2 \pi i} \int_{\partial \tilde{S}_{j}} \frac{\tilde{g} t^{M}}{\tilde{k} t^{m_{j}}} d\left(\frac{\tilde{k} t^{m_{j}}}{\tilde{g} t^{M}}\right) \\
& =\frac{1}{2 \pi i} \int_{\partial \tilde{S}_{j}} \frac{\tilde{g}}{\tilde{k}} d\left(\frac{\tilde{k}}{\tilde{g}}\right)+\left(m_{j}-M\right) \frac{d t}{t} \\
& =\frac{1}{2 \pi i} \int_{\partial S_{j}} \frac{g}{k} d\left(\frac{k}{g}\right)+m_{j}\left(m_{j}-M\right) \\
& =G S V\left(\mathcal{F}, S_{j}, p\right)+m_{j}\left(m_{j}-M\right) .
\end{aligned}
$$

Similarly, if $M_{f}=\operatorname{ord}\left(\mathcal{G}_{f}, p\right)$ :

$$
G S V\left(\tilde{\mathcal{G}}_{f}, \tilde{S}_{j}, q_{j}\right)=G S V\left(\mathcal{G}_{f}, S_{j}, p\right)+m_{j}\left(m_{j}-M_{f}\right) .
$$


But $G S V\left(\tilde{\mathcal{F}}, \tilde{S}_{j}, q_{j}\right) \geq G S V\left(\tilde{\mathcal{G}}_{f}, \tilde{S}_{j}, q_{j}\right)$ by induction hypothesis, and we have proven before that $M \geq M_{f}$, so that

$$
G S V\left(\mathcal{F}, S_{j}, p\right) \geq G S V\left(\mathcal{G}_{f}, S_{j}, p\right)
$$

and using the formula before Proposition 4 we finally obtain the second inequality of the claim.

Let us return to the proof of Proposition 6. We take as $f$ a reduced equation of $S$, then the 1 -form generating $\mathcal{G}_{f}$ is simply $d f$, so that $G S V\left(\mathcal{G}_{f}, S^{\prime}, p\right)=\left(S^{\prime} \cdot S^{\prime \prime}\right)_{p}$ as a simple computation shows. By the previous claim we obtain the desired result:

$$
G S V\left(\mathcal{F}, S^{\prime}, p\right) \geq\left(S^{\prime} \cdot S^{\prime \prime}\right)_{p}
$$

Recall that the singularity of $\mathcal{F}$ at $p$ is said to be a generalized curve if it is nondicritical and there are no saddle-nodes in its resolution [CLS]. By nondicriticalness, the union of all separatrices of a generalized curve is still a separatrix, the "maximal" one. In this case Proposition 6 can be improved

Proposition 7. If the singularity of $\mathcal{F}$ at $p$ is a generalized curve and if $S$ is the union of all separatrices then

$$
G S V(\mathcal{F}, S, p)=0 .
$$

Proof: Using the same notations as in the previous proof, we have now that the inequalities of the claim become equalities [CLS]. In fact, looking at that proof, one sees that the only possibility to have a strict inequality is that $\tilde{\mathcal{G}}_{f}$ has on $D$ less singularities than $\tilde{\mathcal{F}}$, and this cannot happen in our situation [CLS], so we can prove the claim with equalities instead of inequalities. Hence we obtain $G S V\left(\mathcal{F}, S^{\prime}, p\right)=\left(S^{\prime} \cdot S^{\prime \prime}\right)_{p}$ and in particular $G S V(\mathcal{F}, S, p)=0$.

Remark. Suppose that $S$ is a nondicritical separatrix satisfying $G S V(\mathcal{F}, S, p)=0$, then, again, all inequalities appearing in Proposition 6 and its proof must become equalities, because we are in an extremal case. This means that the resolution of $\mathcal{G}_{f}$ is almost a resolution of $\mathcal{F}$. More precisely, if $\pi: \tilde{X} \rightarrow X$ is the desingularization of $S$ which appears in the definition of nondicritical separatrix, then $\pi^{*}(\mathcal{F})$ has only nondegenerate singularities, and all these singularities are in correspondence with the normal crossing points of $\pi^{-1}(S)$. The only difference with the generalized curve case is that some of these singularities can be dicritical (like $n z d w-m w d z=0$, with $n$ and $m$ positive integers). 


\section{The case of almost Liouvillean singularities}

The singularity of $\mathcal{F}$ at $p$ is called almost Liouvillean if there exist a closed meromorphic 1-form $\gamma_{0}$ and a holomorphic 1-form $\gamma_{1}$ near $p$ such that

$$
d \omega=\left(\gamma_{0}+\gamma_{1}\right) \wedge \omega .
$$

If one can choose $\gamma_{1}=0$ then $p$ is called Liouvillean. The polar divisor of $\gamma=\gamma_{1}+\gamma_{0}$ is invariant by $\mathcal{F}$ : near a point $q \in(\gamma)_{\infty} \backslash(p)$ we may choose coordinates $(z, w)$ such that $\omega=a d z, a \in \mathcal{O}^{*}$; if $\gamma_{0}=b d z+c d w$, $b, c$ meromorphic, then $d \gamma_{0}=0$ means $b_{w}=c_{z}$ and $d \omega=\gamma \wedge \omega$ implies that $c$ is holomorphic; hence $c_{z}$ also is holomorphic and consequently the poles of $b$ (i.e. of $\gamma$ ) are invariant by $\mathcal{F}$.

Let $\cup_{j=1}^{N} S_{j}$ be the decomposition of $S=(\gamma)_{\infty}$ into irreducible components. Each $S_{j}$ is a separatrix of $\mathcal{F}$ at $p$; we denote by $\operatorname{Res}\left(\gamma_{0}, S_{j}\right)$ the residue of $\gamma_{0}$ around $S_{j}$. We will say that the almost Liouvillean singularity is simple if one can choose $\gamma_{0}$ having only first order poles.

Proposition 8. If the singularity of $\mathcal{F}$ at $p$ is a simple almost Liouvillean singularity then

$$
B B(\mathcal{F}, p)=\sum_{j=1}^{N} \operatorname{Res}\left(\gamma_{0}, S_{j}\right) \cdot \operatorname{Var}\left(\mathcal{F}, S_{j}, p\right) .
$$

Proof: Let $\gamma^{\prime}$ be a holomorphic 1-form on a tubular neighborhood $V$ of $\partial S$ such that $d \omega=\gamma^{\prime} \wedge \omega$. Let $\phi \in C_{c}^{\infty}(V)$ be equal to 1 on a smaller neighborhood of $\partial S$. Then $\beta=\phi \gamma^{\prime}+(1-\phi) \gamma$ is a smooth $(1,0)$-form on a neighborhood of $\mathbf{S}^{3}$, and $d \omega=\beta \wedge \omega$. A simple computation gives $\beta \wedge d \beta=d \phi \wedge \gamma^{\prime} \wedge \gamma$ and so

$$
\begin{aligned}
\int_{\mathbf{S}^{3}} \beta \wedge d \beta=\int_{\mathbf{S}^{3} \cap V} \beta \wedge d \beta= & \int_{\mathbf{S}^{3} \cap V} d\left((1-\phi) \gamma \wedge \gamma^{\prime}\right) \\
& =\int_{\partial\left(\mathbf{S}^{3} \cap V\right)} \gamma \wedge \gamma^{\prime}=\int_{\partial\left(\mathbf{S}^{3} \cap V\right)} \gamma_{0} \wedge \gamma^{\prime} .
\end{aligned}
$$

We may choose holomorphic coordinates $(z, w)$ near each $\partial S_{j}$, with $z$ varing on a neighborhood of the unit circle and $w$ on a neighborhood of zero, such that $S_{j}=\{w=0\}$ and $\partial S_{j}=\{w=0,|z|=1\}$. Then, on the domain of these coordinates, $\gamma_{0}=\lambda_{j} \frac{d w}{w}+\gamma_{0 j}$, where $\lambda_{j}=\operatorname{Res}\left(\gamma_{0}, S_{j}\right)$ and $\gamma_{0 j}$ is holomorphic, and $\gamma^{\prime}=a d z+b d w$ with $a, b$ holomorphic functions. Moreover, we may assume that the small sphere $\mathbf{S}^{3}$ (which is not necessarily a round sphere) and the neighborhood $V$ are choosen so 
that $V=\cup_{j=1}^{N} V_{j}, V_{j}=$ neighborhood of $\partial S_{j}, \mathbf{S}^{3} \cap V_{j}=\{|w|<\epsilon,|z|=1\}$. Hence the previous integral becomes a sum of terms of the form

$$
\begin{aligned}
\int_{|w|=\epsilon,|z|=1} \lambda_{j} \frac{d w}{w} \wedge a d z= & 2 \pi i \lambda_{j} \int_{|z|=1} a(z, 0) d z \\
& =2 \pi i \lambda_{j} \int_{\partial S_{j}} \gamma^{\prime}=(2 \pi i)^{2} \lambda_{j} \operatorname{Var}\left(\mathcal{F}, S_{j}, p\right)
\end{aligned}
$$

and finally

$$
B B(\mathcal{F}, p)=\sum_{j=1}^{N} \lambda_{j} \operatorname{Var}\left(\mathcal{F}, S_{j}, p\right) .
$$

Example. Let $\mathcal{F}$ be generated by

$$
\omega=z(\lambda+\cdots) d w-w(\mu+\cdots) d z \quad(\lambda, \mu \neq 0)
$$

and let $\alpha, \beta$ be any two complex numbers such that $\alpha \lambda+\beta \mu=\lambda+\mu$. Then if we set $\gamma_{0}=\alpha \frac{d z}{z}+\beta \frac{d w}{w}$, we may find a suitable holomorphic 1-form $\gamma_{1}$ such that $d \omega=\left(\gamma_{0}+\gamma_{1}\right) \wedge \omega$. Let $S_{1}=\{z=0\}, S_{2}=\{w=0\}$, then

$$
\begin{aligned}
& \operatorname{Var}\left(\mathcal{F}, S_{1}, p\right)=\frac{\lambda}{\mu}+1 \\
& \operatorname{Var}\left(\mathcal{F}, S_{2}, p\right)=\frac{\mu}{\lambda}+1
\end{aligned}
$$

and, using Proposition 8,

$$
B B(\mathcal{F}, p)=\alpha\left(\frac{\lambda}{\mu}+1\right)+\beta\left(\frac{\mu}{\lambda}+1\right)=\frac{\lambda}{\mu}+\frac{\mu}{\lambda}+2 .
$$

Example. Let $\mathcal{F}$ be generated by

$$
\omega=z^{p+1} d w-w\left(1+\lambda z^{p}\right) d z, \quad p \geq 1, \lambda \in \mathbf{C}
$$

(the formal normal form of a saddle-node), then $d \omega=\gamma \wedge \omega$ with $\gamma=$ $(p+1) \frac{d z}{z}+\frac{d w}{w}$. If $S_{1}=\{z=0\}, S_{2}=\{w=0\}$, one finds

$$
\begin{gathered}
C S\left(\mathcal{F}, S_{1}, p\right)=0, \quad C S\left(\mathcal{F}, S_{2}, p\right)=\lambda \\
G S V\left(\mathcal{F}, S_{1}, p\right)=1, \quad G S V\left(\mathcal{F}, S_{2}, p\right)=p+1 \\
B B(\mathcal{F}, p)=(p+1)(1+0)+1(p+1+\lambda)=2 p+2+\lambda .
\end{gathered}
$$




\section{The case of generalized curves}

The second statement of the theorem mentioned in the introduction has been proved in Proposition 7. We now prove the first statement, in a form which is a little more general (see the remark after Proposition 7).

Proposition 9. If $S$ is a nondicritical separatrix with the property $G S V(\mathcal{F}, S, p)=0$ then

$$
B B(\mathcal{F}, p)=C S(\mathcal{F}, S, p)
$$

Proof: Let $\pi: \tilde{X} \rightarrow X$ be the desingularization of $S$ at $p$ appearing in the definition of nondicritical separatrix, $D=\cup_{j=1}^{N} D_{j}=\pi^{-1}(p)$ the exceptional divisor. Let $U \subset X$ be a (small) spherical neighborhood of $p$ and $\tilde{U}=\pi^{-1}(U), \tilde{\mathcal{F}}=\pi^{*}\left(\left.\mathcal{F}\right|_{U}\right)$. Let $\omega \in \Omega^{1}(U)$ be a 1-form generating $\mathcal{F}$; the 1 -form $\tilde{\omega}=\pi^{*} \omega$ vanishes on every $D_{j}$, with vanishing order $m_{j}$, so that

$$
c_{1}\left(N_{\tilde{\mathcal{F}}}^{*}\right)=\sum_{j=1}^{N} m_{j}\left[D_{j}\right]
$$

Let $p_{1}, \ldots, p_{M}$ be the singularities of $\tilde{\mathcal{F}}$ which are not corners of $D$, and let $p_{M+1}, \ldots, p_{L}$ be the ones which coincide with corners. We clearly have (see section 1) $B B(\mathcal{F}, p)=B B(\tilde{\mathcal{F}}, \partial \tilde{U})$ and hence, by Proposition 1:

$$
B B(\mathcal{F}, p)=\sum_{j=1}^{L} B B\left(\tilde{\mathcal{F}}, p_{j}\right)-c_{1}^{2}\left(N_{\tilde{\mathcal{F}}}\right) .
$$

Every $p_{j}$ is a nondegenerate singularity with exactly two separatrices contained in $\pi^{-1}(S)$. If $j=1, \ldots, M$ we denote by $S_{j}^{0}$ the separatrix transverse to $D$, which projects by $\pi$ to an irreducible component $\hat{S}_{j}$ of $S$, and by $S_{j}^{1}$ the separatrix contained in $D$. If $j=M+1, \ldots, L$ we denote by $S_{j}^{1}$ the union of the two separatrices, both contained in $D$. By the previous computations:

$$
\begin{array}{ll}
B B\left(\tilde{\mathcal{F}}, p_{j}\right)=\operatorname{Var}\left(\tilde{\mathcal{F}}, S_{j}^{0}, p_{j}\right)+\operatorname{Var}\left(\tilde{\mathcal{F}}, S_{j}^{1}, p_{j}\right) & \text { if } 1 \leq j \leq M \\
B B\left(\tilde{\mathcal{F}}, p_{j}\right)=\operatorname{Var}\left(\tilde{\mathcal{F}}, S_{j}^{1}, p_{j}\right) & \text { if } M+1 \leq j \leq L .
\end{array}
$$

By Proposition 2 the sum of terms $\operatorname{Var}\left(\tilde{\mathcal{F}}, S_{j}^{1}, p_{j}\right)$ is equal to $c_{1}\left(N_{\tilde{\mathcal{F}}}\right) \cdot D$, and so we find

$$
B B(\mathcal{F}, p)=\sum_{j=1}^{M} \operatorname{Var}\left(\tilde{\mathcal{F}}, S_{j}^{0}, p_{j}\right)+c_{1}\left(N_{\tilde{\mathcal{F}}}\right) \cdot D-c_{1}^{2}\left(N_{\tilde{\mathcal{F}}}\right)
$$


We now study the relation between $\operatorname{Var}\left(\tilde{\mathcal{F}}, S_{j}^{0}, p_{j}\right)$ and $\operatorname{Var}\left(\mathcal{F}, \hat{S}_{j}, p\right)$, by an argument similar to that used in Propositions 6 and 7. Let $\omega_{0}$ be a 1-form generating $\tilde{\mathcal{F}}$ near $p_{j}$ and vanishing only at $p_{j}$; hence $\tilde{\omega}=h \omega_{0}$ where $h$ vanishes on $D$ at order $m_{k}, k$ being the index such that $p_{j} \in D_{k}$. Let $\beta$ be a smooth $(1,0)$-form near $\partial \hat{S}_{j}$ such that $d \omega=\beta \wedge \omega$, and let $\tilde{\beta}=\pi^{*} \beta$, so that near $\partial S_{j}^{0}$ we have $d \tilde{\omega}=\tilde{\beta} \wedge \tilde{\omega}$. We obtain, again near $\partial S_{j}^{0}, d \omega_{0}=\left(\tilde{\beta}-\frac{d h}{h}\right) \wedge \omega_{0}$, hence

$$
\begin{aligned}
\operatorname{Var}\left(\tilde{\mathcal{F}}, S_{j}^{0}, p_{j}\right)= & \frac{1}{2 \pi i} \int_{\partial S_{j}^{0}} \tilde{\beta}-\frac{d h}{h} \\
& =\frac{1}{2 \pi i} \int_{\partial \hat{S}_{j}} \beta-\frac{1}{2 \pi i} \int_{\partial S_{j}^{0}} \frac{d h}{h}=\operatorname{Var}\left(\mathcal{F}, \hat{S}_{j}, p\right)-m_{k} .
\end{aligned}
$$

If $l_{k}, k=1, \ldots, N$, is the number of singularities of $\tilde{\mathcal{F}}$ on $D_{k}$ different from corners, we obtain

$$
\begin{aligned}
\sum_{j=1}^{M} \operatorname{Var}\left(\tilde{\mathcal{F}}, S_{j}^{0}, p_{j}\right)=\sum_{j=1}^{M} \operatorname{Var}\left(\mathcal{F}, \hat{S}_{j}, p\right)-\sum_{k=1}^{N} l_{k} m_{k} & \\
& =\operatorname{Var}(\mathcal{F}, S, p)-\sum_{k=1}^{N} l_{k} m_{k}
\end{aligned}
$$

and hence

$$
B B(\mathcal{F}, p)=\operatorname{Var}(\mathcal{F}, S, p)-\sum_{k=1}^{N} l_{k} m_{k}+c_{1}\left(N_{\tilde{\mathcal{F}}}\right) \cdot D-c_{1}^{2}\left(N_{\tilde{\mathcal{F}}}\right) .
$$

On the other hand, the numbers $l_{k}$ can be computed from Proposition 4: if $c_{k}$ is the numbers of corners on $D_{k}$ then

$$
l_{k}+c_{k}=c_{1}\left(N_{\tilde{\mathcal{F}}}\right) \cdot D_{k}-D_{k}^{2}
$$

but $c_{k}=D_{k} \cdot D-D_{k}^{2}$, so that

$$
l_{k}=c_{1}\left(N_{\tilde{\mathcal{F}}}\right) \cdot D_{k}-D \cdot D_{k}=\left(c_{1}\left(N_{\tilde{\mathcal{F}}}\right)-D\right) \cdot D_{k} .
$$

As a consequence, we have

$$
\begin{aligned}
\sum_{k=1}^{N} l_{k} m_{k}=\left(c_{1}\left(N_{\tilde{\mathcal{F}}}\right)-D\right) \cdot \sum_{k=1}^{N} m_{k} D_{k} \\
=\left(c_{1}\left(N_{\tilde{\mathcal{F}}}\right)-D\right) \cdot c_{1}\left(N_{\tilde{\mathcal{F}}}^{*}\right)=c_{1}\left(N_{\tilde{\mathcal{F}}}\right) \cdot D-c_{1}^{2}\left(N_{\tilde{\mathcal{F}}}\right)
\end{aligned}
$$


and finally

$$
B B(\mathcal{F}, p)=\operatorname{Var}(\mathcal{F}, S, p) .
$$

The proof is then achieved by Propositions 5 and 7 .

Remark that by Proposition 5 and the additivity of Var, this result can be reformulated as follows:

$$
B B(\mathcal{F}, p)=\sum_{j=1}^{M}\left[C S\left(\mathcal{F}, S_{j}, p\right)+G S V\left(\mathcal{F}, S_{j}, p\right)\right]
$$

where $\cup_{j=1}^{M} S_{j}$ is any decomposition of $S$ (for example, the decomposition into irreducible components).

\section{Some global remarks}

Let $X$ be a compact surface and let $\mathcal{F}$ be a holomorphic foliation on $X$ leaving invariant a compact curve $S \subset X$. If $S$ is nondicritical (i.e., it is a nondicritical separatrix at every singular point of $\mathcal{F}$ on $S$ ) the Propositions 4 and 6 imply the inequality

$$
S \cdot S \leq c_{1}\left(N_{\mathcal{F}}\right) \cdot S
$$

We study here the limit case $S \cdot S=c_{1}\left(N_{\mathcal{F}}\right) \cdot S$, as it was done (among other things) in $[\mathbf{C L}]$ for the case of a curve in $\mathbf{C} P^{2}$ with only normal crossing singularities.

The first remark is that $c_{1}\left(N_{\mathcal{F}}\right) \cdot S=S \cdot S$ implies that for every irreducible component $S_{j}$ we also have

$$
c_{1}\left(N_{\mathcal{F}}\right) \cdot S_{j}=S \cdot S_{j} .
$$

This holds because for every $p \in \operatorname{Sing}(\mathcal{F}) \cap S$ we have not only $G S V(\mathcal{F}, S, p)=0$ but also $G S V\left(\mathcal{F}, S_{j}, p\right)=\left(S_{j} \cdot \overline{S \backslash S_{j}}\right)_{p}$ (see section 3 ), and hence

$$
\begin{array}{rl}
c_{1}\left(N_{\mathcal{F}}\right) \cdot S_{j}=S_{j} \cdot S_{j}+\sum_{p \in \operatorname{Sing}(\mathcal{F}) \cap S_{j}} & G S V\left(\mathcal{F}, S_{j}, p\right) \\
& =S_{j} \cdot S_{j}+\sum_{p}\left(S_{j} \cdot \overline{S \backslash S_{j}}\right)_{p}=S_{j} \cdot S .
\end{array}
$$

In other words, the line bundle $N_{\mathcal{F}}^{*} \otimes \mathcal{O}(S)$ is topologically trivial on $S$ :

$$
c_{1}\left(N_{\mathcal{F}}^{*} \otimes \mathcal{O}(S)\right) \cdot S_{j}=0 \quad \forall j=1, \ldots, N .
$$


The second remark, contained in $[\mathbf{C L S}]$ when the singularities of $\mathcal{F}$ are generalized curves, is the following. Near $p \in \operatorname{Sing}(\mathcal{F}) \cap S$ choose a holomorphic 1-form $\omega$ generating $\mathcal{F}$ and a reduced equation $f$ of $S$, so that $\frac{\omega}{f}$ has a first order pole on $S$. Let $\pi: \tilde{X} \rightarrow X$ be the resolution of $S$ at $p$ appearing in the definition of nondicriticalness and set $\tilde{\omega}=\pi^{*} \omega, \tilde{f}=$ $f \circ \pi$. Because $G S V(\mathcal{F}, S, p)=0$, we have also $\operatorname{ord}(\mathcal{F}, p)=\operatorname{ord}\left(\mathcal{G}_{f}, p\right)$, that is the vanishing order of $\tilde{f}$ on every component of $\pi^{-1}(p)$ is equal to the vanishing order of $\tilde{\omega}$ plus 1 (this is checked, as usual, by induction). That is, $\frac{\tilde{\omega}}{\tilde{f}}$ has again a first order pole on $\pi^{-1}(S)$. Or, equivalently, $\tilde{S}=$ $\pi^{-1}(S)$ is still a nondicritical $\tilde{F}$-invariant curve satisfying the extremal equality $c_{1}\left(N_{\tilde{\mathcal{F}}}\right) \cdot \tilde{S}=\tilde{S} \cdot \tilde{S}$

After these two remarks, we can state the following proposition, based on a theorem of Deligne $[\mathbf{D e}],[\mathbf{N o g}]$.

Proposition 10. Let $X$ be a compact Kähler surface having the property that the "real Chern class map" $H^{1}\left(X, \mathcal{O}^{*}\right) \stackrel{c}{\rightarrow} H^{2}(X, \mathbf{R})$ is injective. Let $\mathcal{F}$ be a holomorphic foliation on $X$ leaving invariant a nondicritical curve $S$ such that:

i) $c_{1}\left(N_{\mathcal{F}}\right) \cdot S=S \cdot S$

ii) $S \cdot S \leq c_{1}^{2}\left(N_{\mathcal{F}}\right)$

iii) the intersection form of $X$ restricted to the subspace generated by the irreducible components $S_{1}, \ldots, S_{N}$ of $S$ has at least one positive eigenvalue, i.e. there exist $m_{1}, \ldots, m_{N} \in \mathbf{Z}$ such that $\left(\sum m_{j} S_{j}\right)^{2}>0$.

Then $\mathcal{F}$ is generated by a closed meromorphic 1-form $\Omega$, having $S$ as first order polar divisor.

Proof: Let us consider the line bundle $L=N_{\mathcal{F}}^{*} \otimes \mathcal{O}(S)$. By i) and ii):

$$
c_{1}^{2}(L)=c_{1}^{2}\left(N_{\mathcal{F}}\right)+S^{2}-2 c_{1}\left(N_{\mathcal{F}}\right) \cdot S=c_{1}^{2}\left(N_{\mathcal{F}}\right)-S^{2} \geq 0
$$

On the other hand, we have seen that $c_{1}(L) \cdot S_{j}=0$ for every $j$. By iii) and Hodge index theorem [BPV, ch. IV], we must have

$$
c_{1}^{2}(L) \leq 0 \text {. }
$$

Hence the only possibility is $c_{1}^{2}(L)=0$, and, again by Hodge index theorem, the real Chern class of $L$ is zero. The hypothesis on $X$ implies that $L$ is the trivial line bundle, that is $N_{\mathcal{F}}=\mathcal{O}(S)$ and so $\mathcal{F}$ can be generated by a meromorphic 1 -form $\Omega$ such that:

a) the zero divisor $\left(\Omega_{0}\right)$ is empty

b) the polar divisor $(\Omega)_{\infty}$ is $S$, and it has order one. 
It remains to prove that $\Omega$ is closed. Let $\pi: \tilde{X} \rightarrow X$ be the composition of the resolutions of the singular points of $S$, so that $\tilde{S}=\pi^{-1}(S)$ is a normal crossing curve, invariant by $\tilde{\mathcal{F}}=\pi^{-1}(\mathcal{F})$. As remarked before, $\tilde{\Omega}=\pi^{*}(\Omega)$ has on $\tilde{S}$ a first order pole. Because $\tilde{S}$ is invariant, $d \tilde{\Omega}$ has the same property. In other words, $\tilde{\Omega}$ is a so called "logarithmic form", and a theorem of Deligne $[\mathbf{D e}],[\mathbf{N o g}]$ asserts that $\tilde{\Omega}$ is closed. Of course, this means that also $\Omega$ is closed.

Three remarks on hypotheses:

1) the hypothesis on the injectivity of the real Chern class map can be omitted, but then the conclusion must be twisted with a flat line bundle: $\Omega$ will be a closed meromorphic 1 -form with values in a suitable flat line bundle;

2) by Proposition 3 and 9 , the hypothesis i) implies that $S \cdot S=$ $\sum_{p \in \operatorname{Sing}(\mathcal{F}) \cap S} B B(\mathcal{F}, p)$, whereas by Proposition $1 c_{1}^{2}\left(N_{\mathcal{F}}\right)=$ $\sum_{p \in \operatorname{Sing}(\mathcal{F})} B B(\mathcal{F}, p)$. Hence i) implies ii) if $B B(\mathcal{F}, p) \geq 0$ for every singular point outside $S$, and in particular if $S$ contains all the singularities of $\mathcal{F}$;

3) obviously i) implies ii) also if the rank of the Néron-Severi group of $X[\mathbf{B P V}]$ is one.

\section{References}

[BB] P. BAum AND R. Bott, On the zeroes of meromorphic vector fields, Essais en l'honneur de De Rham (1970), 29-47.

[BPV] W. Barth, C. Peters and A. Van de Ven, "Compact complex surfaces," Springer Verlag, 1984.

[Br] M. Brunella, Feuilletages holomorphes sur les surfaces complexes compactes, Prépublication n. 86, Univ. de Dijon (1996).

[CL] D. Cerveau and A. Lins Neto, Holomorphic foliations in $\mathbf{C} P^{2}$ having an invariant algebraic curve, Ann. Inst. Fourier (Grenoble) 41 (1991), 883-903.

[CLS] C. Camacho, A. Lins Neto and P. Sad, Topological invariants and equidesingularization for holomorphic vector fields, $J$. Differential Geom. 20 (1984), 143-174.

[CS] C. CAMACHO AND P. SAD, Invariant varieties through singularities of holomorphic vector fields, Ann. of Math. 115 (1982), 579-595.

[Car] M. CARnicer, The Poincaré problem in the nondicritical case, Ann. of Math. 140 (1994), 289-294.

[De] P. Deligne, Théorie de Hodge II, Publ. IHES 40 (1971), 5-57. 
[GM] X. Gomez-Mont, Universal families of foliations by curves, Astérisque 150-151 (1987), 109-129.

[GSV] X. Gomez-Mont, J. Seade And A. Verjovsky, The index of a holomorphic flow with an isolated singularity, Math. Ann. 291 (1991), 737-751.

[GH] P. GRIfFIths AND J. HarRIs, "Principles of algebraic geometry," Wiley, 1978.

[KS] B. Khanedani and T. Suwa, First variations of holomorphic forms and some applications, Preprint (1996).

[LN] A. Lins Neto, Algebraic solutions of polynomial differential equations and foliations in dimension two, in "Holomorphic Dynamics," (Mexico, 1986), Springer, Lecture Notes 1345, 1988, pp. 192-232.

[Nog] J. Noguchi, A short analytic proof of closedness of logarithmic forms, Kodai Math. J. 18 (1995), 295-299.

[Su T. SuwA, Indices of holomorphic vector fields relative to invariant curves on surfaces, Proc. Amer. Math. Soc. 123 (1995), 2989-2997.

\author{
Laboratoire de Topologie \\ CNRS UMR 5584 \\ Université de Bourgogne \\ B.P. 400 \\ 21011 Dijon \\ FRANCE
}

Primera versió rebuda el 12 de Juliol de 1996, darrera versió rebuda el 17 de Febrer de 1997 